PREPARED FOR THE U.S. DEPARTMENT OF ENERGY, UNDER CONTRACT DE-AC02-76CH03073

PPPL-3646

PPPL-3646

UC-70

Physics Basis and Simulation of Burning Plasma Physics for the Fusion Ignition Research Experiment (FIRE)

by

C.E. Kessel, D. Meade, and S.C. Jardin

January 2002

NM|

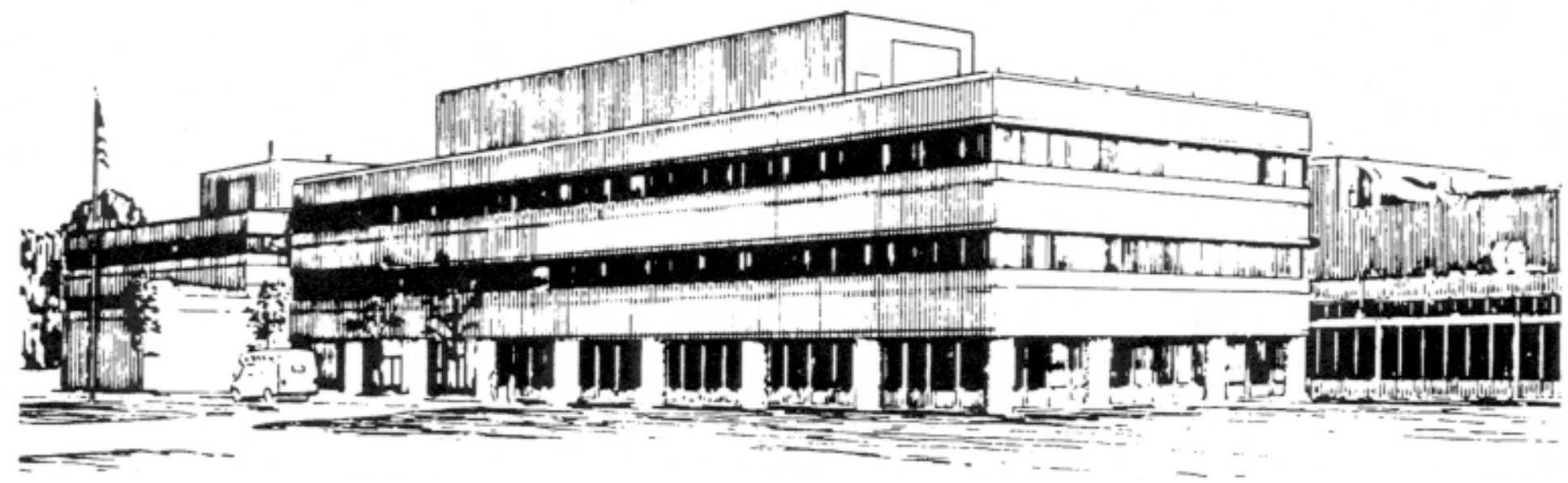

PRINCETON PLASMA PHYSICS LABORATORY PRINCETON UNIVERSITY, PRINCETON, NEW JERSEY 


\section{PPPL Reports Disclaimer}

This report was prepared as an account of work sponsored by an agency of the United States Government. Neither the United States Government nor any agency thereof, nor any of their employees, makes any warranty, express or implied, or assumes any legal liability or responsibility for the accuracy, completeness, or usefulness of any information, apparatus, product, or process disclosed, or represents that its use would not infringe privately owned rights. Reference herein to any specific commercial product, process, or service by trade name, trademark, manufacturer, or otherwise, does not necessarily constitute or imply its endorsement, recommendation, or favoring by the United States Government or any agency thereof. The views and opinions of authors expressed herein do not necessarily state or reflect those of the United States Government or any agency thereof.

\section{Availability}

This report is posted on the U.S. Department of Energy's Princeton Plasma Physics Laboratory Publications and Reports web site in Fiscal Year 2002. The home page for PPPL Reports and Publications is: http://www.pppl.gov/pub_report/

DOE and DOE Contractors can obtain copies of this report from:

U.S. Department of Energy

Office of Scientific and Technical Information

DOE Technical Information Services (DTIS)

P.O. Box 62

Oak Ridge, TN 37831

Telephone: (865) 576-8401

Fax: (865) 576-5728

Email: reports@adonis.osti.gov

This report is available to the general public from:

National Technical Information Service

U.S. Department of Commerce

5285 Port Royal Road

Springfield, VA 22161

Telephone: 1-800-553-6847 or

(703) 605-6000

Fax: (703) 321-8547

Internet: http://www.ntis.gov/ordering.htm 


\title{
Physics Basis and Simulation of Burning Plasma Physics for the Fusion Ignition Research Experiment (FIRE)
}

\author{
C. E. Kessel*, D. Meade, and S. C. Jardin \\ Princeton Plasma Physics Laboratory, P.O. Box 451, Princeton, NJ 08543 \\ *phone 609-243-2294, FAX 609-243-3030, email: ckessel@pppl.gov
}

\begin{abstract}
The FIRE design for a burning plasma experiment is described in terms of its physics basis and engineering features. Systems analysis indicates that the device has a wide operating space to accomplish its mission, both for the ELMing H-mode reference and the high bootstrap current/high $\beta$ advanced tokamak regimes. Simulations with $1.5 \mathrm{D}$ transport codes reported here both confirm and constrain the systems projections. Experimental and theoretical results are used to establish the basis for successful burning plasma experiments in FIRE.
\end{abstract}

\section{Introduction}

The Fusion Ignition Research Experiment (FIRE) is a compact high field copper magnet tokamak design for the study of burning plasma physics [1]. Its primary goal is to explore the physics of self-heated plasmas by obtaining a fusion gain Q(Pfus/Paux) 10 with flattop times greater than 1.5 times the current redistribution time, and a cost target of $\sim \$ 1 B$. Inductively driven plasmas with ELMing (edge localized mode) H-mode energy confinement is the reference operating regime and this is used as the basis for projecting the global energy confinement time [2]. The device is also capable of examining advanced tokamak (AT) regimes with additional heating/current drive [3]. The main plasma parameters are $\mathrm{Ip}=7.7 \mathrm{MA}, \mathrm{Bt}=10 \mathrm{~T}, \mathrm{R}=2.14 \mathrm{~m}, \mathrm{a}=0.595 \mathrm{~m}, \mathrm{\kappa}=2.0$, $\delta=0.7$, and q95>3.0. Additional plasma parameters are listed in Table 1 .

\section{Physics Basis and Engineering Features}

Strong plasma shaping is pursued with a separatrix elongation of 2.0 and triangularity of 0.7 , with double null (DN) operation. The triangularity is limited by the distance between the $\mathrm{x}$-point and strike point on the inboard side to provide sufficient distance for power dissipation. Calculations indicate that the inboard should detach [4] over a wide range of parameters. The high triangularity provides higher ideal MHD beta limits, and leads to higher pedestal pressures [5-7] and access to higher plasma densities relative to the Greenwald density limit [7] with no energy confinement degradation. Although higher triangularity has led to less frequent, and therefore stronger, ELMs [8,9], recent data with a pumped divertor indicates that this is not necessarily the case [10]. The plasma elongation is limited by the $\mathrm{n}=0$ axisymmetric (or vertical) instability. Copper stabilizing plates, $1.5 \mathrm{~cm}$ thick, are joined to the inner shell of the stainless steel vacuum vessel (VV) in the upper outboard and inboard to provide passive stabilization. The growth times, at $\beta \mathrm{p}=0.1$, for the instability are between 19 and $43 \mathrm{~ms}$ for li (plasma internal self-inductance) values between 1.1 and 0.7 , respectively. These lead to stability factors $(1+\tau \mathrm{g} / \tau \mathrm{L} / \mathrm{R})$ between 1.15 and 1.3 . Vertical position control is provided by a pair of coils located behind the inner VV wall, but inside the outer VV and TF coils. The peak feedback control power requirement is in the range of 6-12 MVA. 
The assumed lower limit on the plasma safety factor at the $95 \%$ flux surface is 3.0 for reliable (nondisruptive) plasma operation. It has been suggested that reliable operation with q95 below 3.0 is possible [11]. This has not been examined in detail for FIRE.

The reference operating point for FIRE has a $\beta \mathrm{N}$ value of 1.6, to be below the expected neoclassical tearing mode (NTM) limit, which is in the range of 2-2.5. The precise condition for these modes to become unstable in the burning plasma regime is uncertain, and so the FIRE design has chosen to operate at low $\beta N$. Initial simulations of stabilization of the $(3,2)$ island with lower hybrid current drive (LHCD) for FIRE appear promising, and both experimental [12] and theoretical [13] evidence indicate this should be possible by locally altering the current profile to make $\Delta^{\prime}$ more negative. LHCD is considered an upgrade for FIRE, and is why a low operating $\beta \mathrm{N}$ is used. The projected ideal MHD limit with no wall stabilization, for $n=\infty$ ballooning and $n=1$ external kink instabilites, show the maximum $\beta \mathrm{N}$ around 3.0, including the proper relaxed ohmic current profiles with self-consistent bootstrap current. Higher $\beta \mathrm{N}$ values can be achieved with feedback and/or plasma rotation stabilization [14,15], and are important for advanced tokamak operating modes to be discussed later.

The plasma impurity assumption for the FIRE design is $3 \% \mathrm{Be}$, which combined with $\mathrm{He}$ ash gives a Zeff of 1.4. Beryllium is the plasma facing material for the first wall. The projected impurity concentration [16] for the high plasma densities in FIRE is less than $0.4 \%$ for Be. Since noble gases have been shown experimentally to be good candidates (Ne and Ar) for controlling plasma radiation, the extra $2.6 \% \mathrm{Be}$ is intended to account for this. This is equivalent to $0.1 \% \mathrm{Ar}$, which is found to double the radiated power from the plasma [17]. The helium concentration must be found from self-consistent power and particle balance, and is about $2 \%$. This assumes a ratio of effective particle confinement time to energy confinement time, $\tau(\mathrm{He}) * / \tau(\mathrm{E})$, of 5.0, which has been achieved on pumped divertor experiments [18].

The plasma density and its profile are critical parameters in burning plasmas since they strongly impact the plasma power balance. The peak plasma density in FIRE, for the reference operating mode, is $5.3 \times 10^{\wedge} 20 / \mathrm{m}^{\wedge} 3$, and the assumed peak to volume average density is 1.2. The peaking is expected from pellet fueling from the inboard and vertical directions, based on experiments showing significantly deeper penetration compared to outboard pellet launch $[19,20]$. The density value at the plasma separatrix is taken to be 0.3 times the peak value, giving $1.6 \times 10^{\wedge} 20 / \mathrm{m}^{\wedge} 3$. The combination of pellet fueling and density peaking are also observed experimentally to be a primary method to approach or exceed the Greenwald density limit without energy confinement degradation. The FIRE reference density divided by the Greenwald density $(\mathrm{n} / \mathrm{nGr})$ is 0.65 , which is well supported in the energy confinement database, and is not expected to result in confinement degradation regardless of the actual density peaking.

Since the projections of global energy confinement time for the design of burning plasma experiments are uncertain, a database of a large number plasma discharges from tokamak experiments has been established to generate scaling relations, which has produced both a global energy confinement time expression for type I ELMing H-mode plasmas [2] and an H-mode power threshold expression [21]. The ratio of the power lost through the plasma boundary to the threshold power to enter the H-mode, $\mathrm{P}(\operatorname{loss}) / \mathrm{P}(\mathrm{LH})$, is 1.3 for the reference operating mode in FIRE, with the threshold power being $25 \mathrm{MW}$. The plasma typically enters the H-mode earlier in the discharge during the rampup phase, but some margin to the threshold may be desirable during the flattop burning phase. Recent experiments show that a number of variations of the H-mode and ELM behavior outside 
of the standard model can be established with desirable properties [10,22-24], and it is clear that some form of low amplitude ELMs are necessary to maximize divertor lifetime. Previously the threshold power was reported to be higher for DN plasmas versus single null (SN) plasmas, however, the most recent evidence from DIII-D indicates that the threshold power for DN is the same or less than that for SN [25]. The energy confinement time scaling law that has been applied to ITER, designated by IPB98(y,2), estimates a global energy confinment time of $0.93 \mathrm{~s}$ for FIRE. The proposed value for the device is $1.0 \mathrm{~s}$ based on a recent examination of parameters not included in the scaling expression [26], in particular, triangularity, proximity to the Greenwald density limit, and density profile peaking. The correction to the scaling law gives an additional factor of 1.07 for FIRE, which recovers the $\tau(E)$ value of $1.0 \mathrm{~s}$.

The plasma auxiliary systems include diagnostics for plasma control and analysis [27]. The heating for FIRE reference operation is provided by $20 \mathrm{MW}$ of ion cyclotron (ICRF) in the frequency range of $80-120 \mathrm{MHz}$, utilizing 4 ports. Port space is alloted for an additional $10 \mathrm{MW}$ upgrade if required. A total of $20 \mathrm{MW}$ of LHCD is reserved as an upgrade for advanced tokamak (AT) operation and NTM control. Plasma fueling is provided by gas injection and pellet fueling, including high field side, vertical and low field side launch. Divertor pumping is achieved with 16 cryopumps, 8 above and 8 below the midplane, in slanted ports behind the outboard divertor modules. These pumps are backed up by turbo/drag pumps outside the biological shield. The predicted peak heat flux on the divertor ranges from 5 to $25 \mathrm{MW} / \mathrm{m}^{\wedge} 2$ [4], the lower values corresponding to detached and the higher to attached operation. The plasma facing surface in the divertor is tungsten bonded to a $\mathrm{CuCrZr}$ heat sink. All other plasma facing surfaces are beryllium coated copper.

The reference flattop is $20 \mathrm{~s}$, with a $7 \mathrm{~s}$ plasma current rampup, and a $7 \mathrm{~s}$ rampdown. This flattop provides approximately 2 current relaxation times, 4 effective helium confinement times, and 20 energy confinement times. The flattop is limited by the toroidal field (TF) coil heating, vacuum vessel nuclear heating which causes a stress limit, and surface and nuclear heating of the first wall tiles. The TF coils can provide 10 $\mathrm{T}$ for $20 \mathrm{~s}, 9.5 \mathrm{~T}$ for $26 \mathrm{~s}$, and $8.5 \mathrm{~T}$ for $35 \mathrm{~s}$, and so on. The VV nuclear heating limits the flattop to $20 \mathrm{~s}$ at a fusion power of $200 \mathrm{MW}$. Finally, the FW tile heating, with conservative assumptions of $120 \%$ radiated power, is not limiting until flattop times of more than $50 \mathrm{~s}$. The neutron wall loading is $2.0 \mathrm{MW} / \mathrm{m}^{\wedge} 2$ at $150 \mathrm{MW}$ of fusion power. The poloidal field (PF) coils can provide both inductive current drive and plasma equilibrium, over a range of li, $\beta$ p, and flux state. Stress limits determine the maximum volt-seconds obtainable from the PF coils, in particular, the central solenoid coils, which is approximately $55 \mathrm{~V}$-s. Radial position and shape control are provided by the PF coils, while vertical position control is provided by an internal control pair.

The maximum TF coil ripple at the outboard midplane is $0.3 \%$ since the outboard legs of the coil are far from the plasma. This results in a total ripple loss, collisionless stochastic plus collisional, of $0.3 \%$. For the advanced tokamak the losses are larger due to higher safety factors. Two cases were examined to bracket the possible range, one with $\mathrm{Ip}=5.7$ $\mathrm{MA}$ and $\mathrm{Bt}=9.0 \mathrm{~T}$ and the second with $\mathrm{Ip}=4.5 \mathrm{MA}$ and $\mathrm{Bt}=6.75 \mathrm{~T}$, both with $\mathrm{q}(0)=3.0$ and $\mathrm{q}(\mathrm{min})=2.5$. The first case yielded a total loss of $2.4 \%$ while the second gave a loss of 7.7\%. Alfven eigenmodes and energetic particle modes are potential instabilities that can lead to alpha particle loss or broadening of the alpha heating profile. This is presently being studied for FIRE.

\section{Systems Analysis of Operating Regions}


OD systems calculations have been used to assess the operating range of FIRE within various physics and engineering constraints. The systems analysis contains plasma power balance, particle balance, and flux consumption combined with several physics expressions. In addition, the temperature and stress on the $\mathrm{TF}$ and $\mathrm{OH}$ coils can be included to solve for the device radial build as the plasma shape and aspect ratio are varied. The determination of optimum aspect ratio for the inductive operating mode, that which gives the minimum major radius, gives a broad minimum between 3.5 and 3.8, for cryogenically cooled copper TF and PF coils used in FIRE. From this analysis the major radius for FIRE is chosen to be $2.14 \mathrm{~m}$ at an aspect ratio of 3.6.

With the device geometry fixed the operating space can be determined by varying the $\mathrm{Q}$, $\mathrm{n} / \mathrm{nGr}$, and fusion power. The $\operatorname{IPB}(\mathrm{y}, 2)$ global energy scaling has been used to determine the value of $\mathrm{H} 98(\mathrm{y}, 2)$ required for power balance, and various assumptions for other parameters are in Table 1. Shown in Fig. 1 is the fusion power versus the H98(y,2) factor required for power balance, with $Q$ values ranging from 5 to 20. Also shown are constant $\mathrm{n} / \mathrm{nGr}$ contours for $\mathrm{Q}=10$. The solid curves for the various $\mathrm{Q}$ values correspond to $\mathrm{n} / \mathrm{nGr}=0.8$. The operating region is cutoff in the vertical direction by either the maximum auxiliary power exceeding $30 \mathrm{MW}$ or $\beta \mathrm{N}$ exceeding 2.5. The lower boundary corresponds to a $\mathrm{P}(\mathrm{loss}) / \mathrm{P}(\mathrm{LH})$ of 1.0. The reference operating point is noted and lies near the boundary for $\mathrm{Q}=10$ operation because the contours of $\mathrm{n} / \mathrm{nGr}$ are crowding together there. Due to the high sensitiviy of Q to the H98 factor, minor improvements in the confinement allow high Q values, shown by the closely nested contours. Also noted is the $200 \mathrm{MW}$ fusion power where the flattop time is $20 \mathrm{~s}$, higher fusion powers would require shorter flattop times. The density and temperature profiles, and the energy confinement scaling strongly affect the operating space.

To determine the operating space for AT plasmas, again the major and minor radius, and elongation, triangularity and aspect ratio are fixed. These have been set by the reference ELMy H-mode inductively driven design point. An expression for the bootstrap current fraction and current drive power is included. A large number of plasma configurations are generated by varying the toroidal field (from 6.5-9.5 T), q95 (from 3.1-4.7), peak to average density (from 1.25 to 2.0 ), $\beta \mathrm{N}$ (from $2.5-4.5$ ), and $\mathrm{n} / \mathrm{nGr}$ (from $0.45-0.85$ ). The acceptable solutions are constrained to have a given $Q$ value, the external current drive power must be less than the total auxiliary power injected into the plasma, and the fusion power must be less than $250 \mathrm{MW}$. The current drive efficiency used in these scans is $\eta \mathrm{cd}=0.45 \mathrm{~A} / \mathrm{W}-\mathrm{m} 2$ and is based on detailed $\mathrm{LH}$ and ICRF/FW analysis for FIRE. Shown in Fig. 2 is the current drive power as a function of the H98 $(y, 2)$ factor, for specific values of $\mathrm{Bt}=8.5 \mathrm{~T}, \beta \mathrm{N}=3.0$, and $\mathrm{Q}=5$. The plot shows curves of constant $\mathrm{q} 95$, which are also constant bootstrap current fraction. In addition, curves for different values of the peak to average density are shown. The major conclusions from this analysis are that $\mathrm{Q}=5$ requires $\mathrm{H} 98(\mathrm{y}, 2)$ factors greater than 1.2 . The peaking of the density is a high leverage quantity both for plasma power balance and for increasing the bootstrap current fraction. Although higher $\mathrm{n} / \mathrm{nGr}$ improves power balance allowing lower H98(y,2) factors, it increases the current drive power, since noninductive sources of current are more efficient at high temperature and low density. In fact, the lowest H98(y,2) factors correspond to $\mathrm{n} / \mathrm{nGr}=0.95$ and $\mathrm{n}(0) /\langle\mathrm{n}\rangle=2.0$ while the current drive power is near its maximum. It should be noted that the $\operatorname{IPB}(y, 2)$ scaling penalizes high $\beta \mathrm{N}$, and it is not clear that this is supported by the experimental data for any particular device.

\section{Simulation of the Reference Operating Scenario}

The Tokamak Simulation Code[28] (TSC) is used to provide the 1.5D simulation of the FIRE reference discharge. Here the plasma density and its profile are specified, while the 
energy transport is modeled with GLF23. Shown in Fig. 3 are some time histories, in Fig. 4 are the various powers into the plasma, and in Fig. 5 are the flattop profiles of density, temperature, and current density. The plasma current is ramped up in $7 \mathrm{~s}$, which is sufficiently slow to allow the sawtooth to relax to its final radius of $0.2 \mathrm{~m}$ before heating is applied. The plasma is grown off the inboard limiter and reaches full size by about $4 \mathrm{~s}$. The ohmic heating reaches just over $5 \mathrm{MW}$ before $20 \mathrm{MW}$ of ICRF power is injected at $4.8 \mathrm{~s}$. The plasma enters H-mode, and then the density is ramped up faster to its final value by $9 \mathrm{~s}$. The electron and ion peak temperatures reach about $4 \mathrm{keV}$ from the ohmic heating alone, and then rise to about $11 \mathrm{keV}$ when the auxiliary heating begins. The plasma breakdown consumes $2 \mathrm{~V}$-s, the rampup consumes $40 \mathrm{~V}-\mathrm{s}$, and the $20 \mathrm{~s}$ flattop consumes $3.7 \mathrm{~V}$-s. A feedback system is used to control the plasma stored energy by adjusting the auxiliary power. This causes the auxiliary power to drop after about $9 \mathrm{~s}$, when the stored energy has reached its final value $34.5 \mathrm{MJ}$, to about $12.5 \mathrm{MW}$. The global energy confinement time is $1.0 \mathrm{~s}$ in flattop. The alpha power is $30 \mathrm{MW}$, the ohmic power is $1.5 \mathrm{MW}$, and the bremsstrahlung loss is $9.6 \mathrm{MW}$. The peak density is $5.3 \times 10^{\wedge} 20 / \mathrm{m}^{\wedge} 3$, the peak temperature is $11.6 \mathrm{keV}$ with sawtooth flattening, and 16.5 $\mathrm{keV}$ without flattening. The Zeff reaches its peak value of 1.4 with $3 \%$ Be impurity, the bootstrap fraction reaches 0.18 , and the volume average He density is $8.0 \times 10^{\wedge} 18 / \mathrm{m}^{\wedge} 3$ with $\tau(\mathrm{He})^{*} / \tau(\mathrm{E})$ assumed to be 5.0. The $\beta \mathrm{N}$ reaches 1.63 , li is $0.77, \beta \mathrm{p}$ is 0.8 , and $\mathrm{n} / \mathrm{nGr}$ is 0.67 . This simulation resulted in a $\mathrm{Q}$ of 12 , however, the energy transport model is quite sensitive to the pedestal temperature, which here was adjusted to recover the $\operatorname{IPB}(y, 2)$ scaling, with a factor of 1.07 , and had the value of $3.7 \mathrm{keV}$.

\section{Simulation of an Advanced Tokamak Scenario}

Equilibrium and ideal MHD stability analysis, combined with LHCD calculations, identified attractive targets for advanced tokamak operation, the most attractive having $\mathrm{q}(\mathrm{min})$ just above 2.0 and $\mathrm{r} / \mathrm{a}(\mathrm{qmin})=0.8$. The low order NTMs could be avoided, although the $(5,2)$ and $(3,1)$ surfaces will be present. The location of $q(\min )$ is determined by the penetration of the LH waves for expected FIRE parameters. On axis current drive requirements from ICRF/FW are typically less than $0.4 \mathrm{MA}$. The maximum $\beta N$, determined for $n=\infty$ ballooning and $n=1$ external kink modes, with no wall stabilization is 2.5 . For a perfectly conducting wall located at the VV location the $\beta \mathrm{N}$ for the $n=1$ mode could rise to 4.6. Calculations performed with the VALEN [30] code using feedback control coils located in the ports indicate that $\beta \mathrm{N}$ can reach 3.8.

TSC is used to simulate the AT discharge, with the LSC [29] ray tracing package connected for the lower hybrid current drive calculations. The fast wave is not calculated self-consistently, but modelled as a prescribed profile and current drive efficiency. The primary goal is to establish quasi-stationary burning plasmas for the flattop, where the current and safety factor profiles do not significantly change. Although inductive and non-inductive current drive are used to ramp the plasma current up, the flattop plasma has $100 \%$ non-inductive current provided by the combination of bootstrap, lower hybrid, and fast wave current.

The parameters for this simulation are $\mathrm{Bt}=8.5 \mathrm{~T}, \mathrm{Ip}=5.4 \mathrm{MA}, \beta \mathrm{N}=3.5, \beta=4.5 \%$, $\mathrm{I}(\mathrm{BS})=3.6 \mathrm{MA}, \mathrm{I}(\mathrm{LH})=1.5 \mathrm{MA}, \mathrm{I}(\mathrm{FW})=0.35 \mathrm{MA}$, and $\mathrm{Q}=7.8$ with $\mathrm{H} 98(\mathrm{y}, 2)=1.6$. Shown in Figs. 6 and 7 are the plasma current density and safety factor, and the currents and powers driven in the plasma, respectively. The plasma current is ramped up over $10 \mathrm{~s}$, and the flattop is $32 \mathrm{~s}$ long. Shown in Fig. 6 are some time histories, and in Fig. 7 are the flattop current density and safety factor profiles. A maximum of $15 \mathrm{MW}$ of ICRF power, to drive the small on axis current and heat the plasma, is injected during the rampup, and dropped to $7 \mathrm{MW}$ in the flattop. The $\mathrm{LH}$ power increases to $20 \mathrm{MW}$ during rampup and 
remains there for the flattop. This provides both off-axis current drive and heating to electrons. The density relative to Greenwald density reaches 0.5 , with the peak density reaching $4.7 \times 10^{\wedge} 20 / \mathrm{m}^{\wedge} 3$, and with a peak to average density of 1.6 . The energy confinement time in flattop is $0.6 \mathrm{~s}$, which is 1.6 times the IPB98 $(\mathrm{y}, 2)$ scaling. The peak electron temperature reaches $24 \mathrm{keV}$, while that for the ions is $20 \mathrm{keV}$, and the peak to average temperatures for both species is 2.0. About $22 \mathrm{~V}$-s were used in the plasma current rampup, which is about 55\% of that required to ramp to the reference current inductively. The flattop alpha power was $42 \mathrm{MW}$. The bremsstrahlung radiation loss was $6.6 \mathrm{MW}$. The impurity is taken to be $3 \%$ Be, which resulted in a Zeff of 1.41 with the He included. The volume average He density was $1.82 \times 10^{\wedge} 19 / \mathrm{m}^{\wedge} 3$. The bootstrap current fraction is $66 \%$, with LH providing $28 \%$ and FW the remaining $6 \%$. The high bootstrap fraction is due to a $\beta \mathrm{N}$ of 3.5 in combination with a stronger density peaking than is typical of standard ELMy H-modes. Although pellet fueling might provide some peaking, it is expected that the formation of an internal transport barrier will provide more significant peaking. Transport calculations to predict the formations of an ITB are underway. The density peaking is also important for efficient LH current drive, whose efficiency scales as T/n, by keeping the density lower in the deposition region.

\section{Conclusions}

The FIRE design with copper TF and PF magnets can provide a compact device for the study of burning plasma physics. The operating space for FIRE allows the study of burning plasmas at various $Q$ values, for time scales greater than the current redistribution time, for an inductively driven plasma in the ELMing H-mode regime. A combination of experimental data on present tokamaks and theoretical modeling and simulation is being used to establish the basis for a successful experiment. In addition, the device has the capability to access a significant operating space for burning plasmas in the high bootstrap current/high $\beta$ advanced tokamak regime. This is accomplished by varying the toroidal field and plasma current, and supplying LH and ICRF/FW current drive. Successful AT experiments in FIRE will rely on experimental and theoretical development in the areas of internal transport barriers, feedback stabilization of the $n=1$ kink mode, and plasma edge control in present tokamaks.

\section{References}

[1] D. Meade, this conference.

[2] ITER Physics Expert Groups, Plasma confinement and transport, Nucl. Fusion, 39 (1999) 2137.

[3] C. E. Kessel, D. Ignat, and T. K. Mau, Advanced tokamak scenarios for the FIRE burning plasma experiment, 19 ${ }^{\text {th }}$ Symp. Fus. Engineering, January 22-25, 2002.

[4] M. Ulrickson, et al., Design of Fusion Ignition Research Experiment (FIRE) plasma facing components, 19 ${ }^{\text {th }}$ Symp. Fus. Engineering, January 22-25, 2002.

[5] A.E. Hubbard, Physics and scaling of the H-mode pedestal, Plasma Phys. Control. Fusion, 42 (2000) A15.

[6] T. Hatae, et al., Understanding of H-mode pedestal characteristics using the multimachine pedestal database, Nucl. Fusion, 41 (2001) 285. 
[7] T. H. Osborne, et al., The effect of plasma shape on H-mode pedestal characteristics on DIII-D, Plasma Phys. Control. Fusion, 42 (2000) A175.

[8] W. Suttrop, The physics of large and small edge localized modes, Plasma Phys. Control. Fusion, 42 (2000) A1.

[9] L. D. Horton, H-mode operational boundaries, Plasma Phys. Control. Fusion, 42 (2000) A37.

[10] Y. Kamada, et al., Disappearance of giant ELMs and appearance of minute grassy ELMs in JT-60U high-triangularity discharges, Plasma Phys. Control. Fusion, 42 (2000) A247.

[11] D. J. Campbell, The physics of the International Thermocuclear Experimental Reactor FEAT, Phys. Plasmas, 8 (2001) 2041.

[12] C. D. Warrick, Complete stabilization of neoclassical tearing modes with lower hybrid current drive on Compass-D, Phys. Rev. Lett., 85 (2000) 574.

[13] A. Pletzer and F. W. Perkins, Stabilization of neoclassical tearing modes using a continuous localized current drive, Phys. Plasmas, 6 (1999) 1589.

[14] A. Bondeson et al., Active feedback stabilization of high beta modes in advanced tokamaks, Nucl. Fusion, 41 (2001) 455.

[15] A. Garofalo, et al., Stabilization of the external kink and control of the resistive wall mode in tokamaks, Phys. Plasmas, 6 (1999) 1893.

[16] G. F. Mathews, et al., Scaling radiative plasmas to ITER, J. Nucl. Mater.., 241-243 (1997) 450.

[17] J. Mandrekas, private communication.

[18] ITER Physics Expert Groups, Power and particle control, Nucl. Fusion, 39 (1999) 2391.

[19] L. R. Baylor, et al., Improved core fueling with high field side pellet injection in the DIII-D tokamak, Phys. Plasmas, 7 (2000) 1878.

[20] P. T. Lang, et al., High-density H-mode operation achieved using efficient plasma refueling by inboard pellet launch, J. Nucl. Mater., 290-293 (2001) 374.

[21] J. A. Snipes, et al., Latest results on the H-mode threshold using the international Hmode threshold database, Plasma Phys. Control. Fusion, 42 (2000) A299.

[22] M. Greenwald, et al., Studies of EDA H-mode in Alcator C-Mod, Plasma Phys. Control. Fusion, 41 (2000) A263.

[23] J. Stober, et al., Type II ELMy H modes on ASDEX Upgrade with good confinement at high density, Nucl. Fusion, 41 (2001) 1123.

[24] K. H. Burrell, et al., Constant density, ELM-free, steady state divertor plasmas in DIII-D, Phys. Plasmas, 8 (2001) 2153. 
[25] T. N. Carlstrom, R. J. Groebner, and T. L. Rhodes, Dependence of the H-mode power threshold on plasma shape in DIII-D, presented at Americn Physical Society Division of Plasma Physics Meeting, October, 2001.

[26] J. G. Cordey, et al., Energy confinement in steady state ELMy H-modes in JET, Proc. $28^{\text {th }}$ EPS Conference on Controlled Fusion and Plasma Physics, 2001, paper 3.11.

[27] K. Young, et al., Challenges for plasma diagnostics in a next step device (FIRE), $19^{\text {th }}$ Symp. Fus. Engineering, January 22-25, 2002.

[28] S. C. Jardin, N. Pomphrey, and J. Delucia, Dynamic modeling of transport and position control of tokamaks, J. Comput. Phys., 66 (1986) 481.

[29] D. Ignat, E. Valeo, and S. C. Jardin, Dynamic modeling of lower hybrid current drive, Nucl. Fusion, 34 (1994) 837.

[30] J. Bialek, A. H. Boozer, M. E. Mauel, and G. A. Navratril, Modeling of active control of external magnetohydrodynamic instabilities, Phys. Plasmas, 8 (2001)

2170 . 


\begin{tabular}{|c|c|}
\hline Parameter & \begin{tabular}{|l|} 
FIRE \\
\end{tabular} \\
\hline $\mathrm{R}, \mathrm{a}(\mathrm{m})$ & $2.14,0.595$ \\
\hline Ip (MA) & 7.7 \\
\hline $\mathrm{Bt}(\mathrm{T})$ & 10.0 \\
\hline$\kappa 95, \kappa(X)$ & $1.77,2.0$ \\
\hline$\delta 95, \delta(X)$ & $0.4,0.7$ \\
\hline $\mathrm{g95}$ & 3.1 \\
\hline $\mathrm{t}$ (flattop), $\tau$ (skin) $(\mathrm{s})$ & $20.0,9.9$ \\
\hline $\mathrm{n} / \mathrm{nGr}$ & 0.65 \\
\hline $\mathrm{n}(0) /<\mathrm{n}>$ & 1.2 \\
\hline Te,i(0), <Te,i> (keV) (pre-sawtooth) & $16.5,6.0$ \\
\hline Te,i(0), <Te,i> (keV) (post-sawtooth) & $12.0,6.0$ \\
\hline$\overline{\text { Zeff }}$ & 1.4 \\
\hline$\overline{\beta N}$ & 1.6 \\
\hline $\mathrm{P}$ (fusion) (MW) & 150.0 \\
\hline $\mathrm{Q}$ & 10.0 \\
\hline$\tau(\mathrm{He})^{*} / \tau(\mathrm{E})$ & 5.0 \\
\hline$\overline{\tau(\mathrm{E}), \mathrm{H} 98(\mathrm{y}, 2)(\mathrm{s})}$ & $1.0,1.07$ \\
\hline $\mathrm{V}$ (loop) (V) & 0.185 \\
\hline li(3) & 0.77 \\
\hline Wth (MJ) & 34.5 \\
\hline$\beta p$ & 0.8 \\
\hline Area(cross-section) $\left(\mathrm{m}^{\wedge} 2\right)$ & 2.06 \\
\hline$\alpha \mathrm{N}, \alpha \mathrm{T}(0 \mathrm{D}$ analysis) & $0.2,1.35$ \\
\hline
\end{tabular}




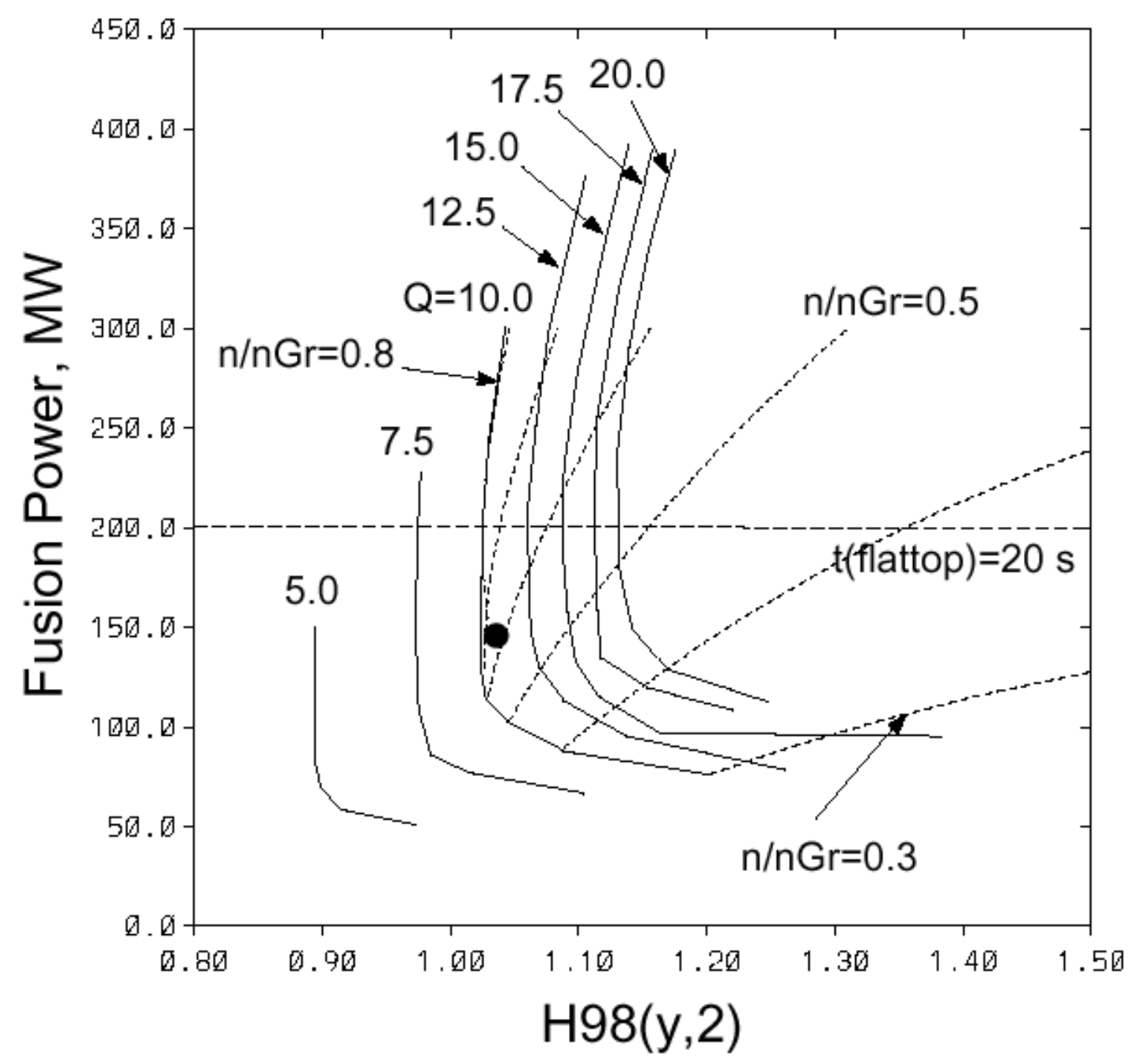

Figure 1: Operating space for FIRE shown as $\mathrm{P}($ fusion) versus $\mathrm{H} 98(\mathrm{y}, 2)$, where solid contours correspond to specific $Q$ values at $n / n G r=0.8$, and the operating space for that $\mathrm{Q}$ lies to the right of the contour. The dashed lines are constant $\mathrm{n} / \mathrm{nGr}$ contours for $\mathrm{Q}=10$. 


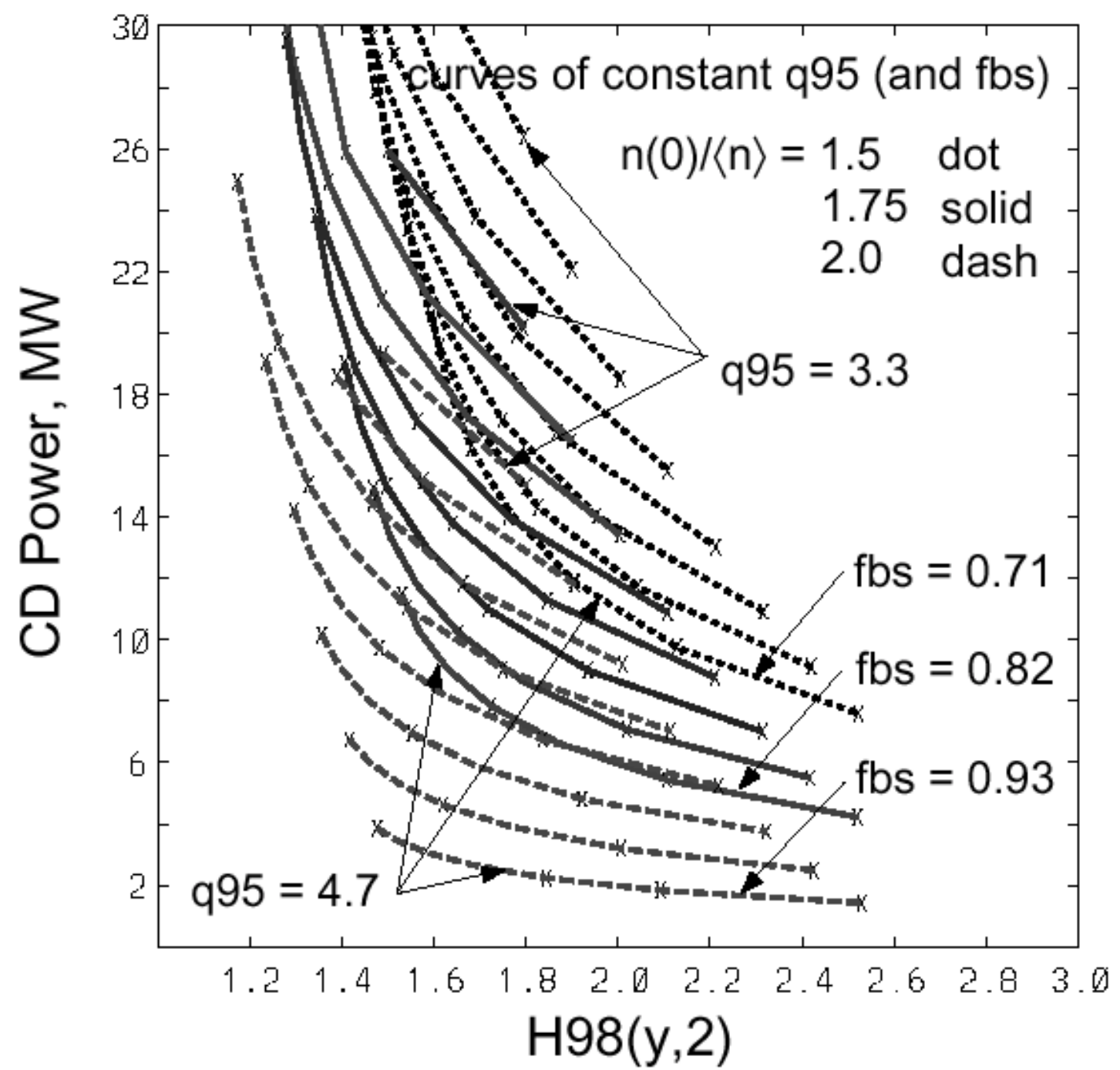

Figure 2: Subset of operating space for FIRE AT plasmas, with $\mathrm{Bt}=8.5 \mathrm{~T}$, $\beta \mathrm{N}=3.0$, and $\mathrm{Q}=5$, shown as current drive power versus $\mathrm{H} 98(\mathrm{y}, 2)$. Contours are constant q95 (and bootstrap fraction), and different peak to volume average density values are given. 

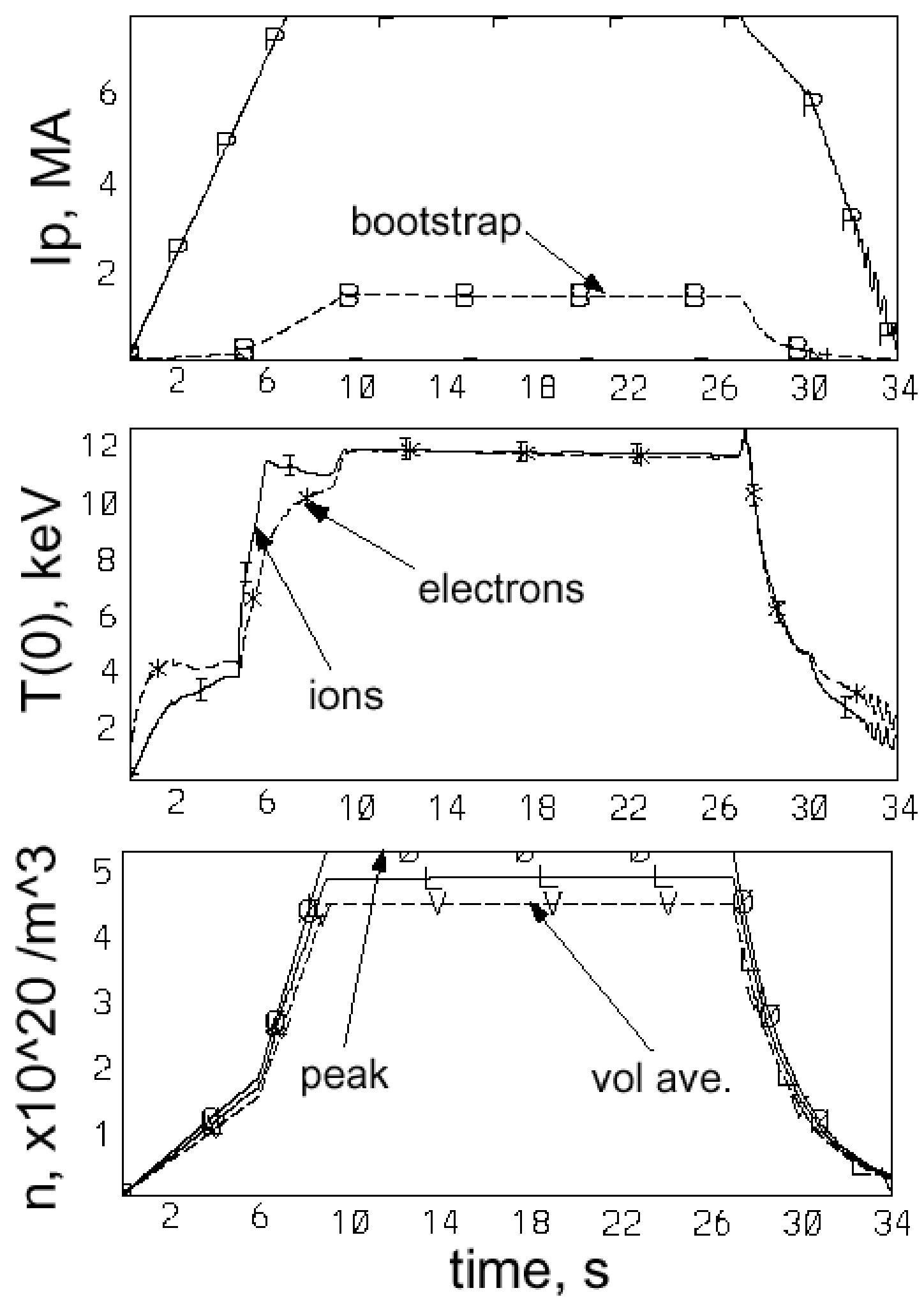

Figure 3: Time histories of the plasma current and bootstrap current, peak electron and ion temperatures, and electron density for the FIRE reference discharge. 


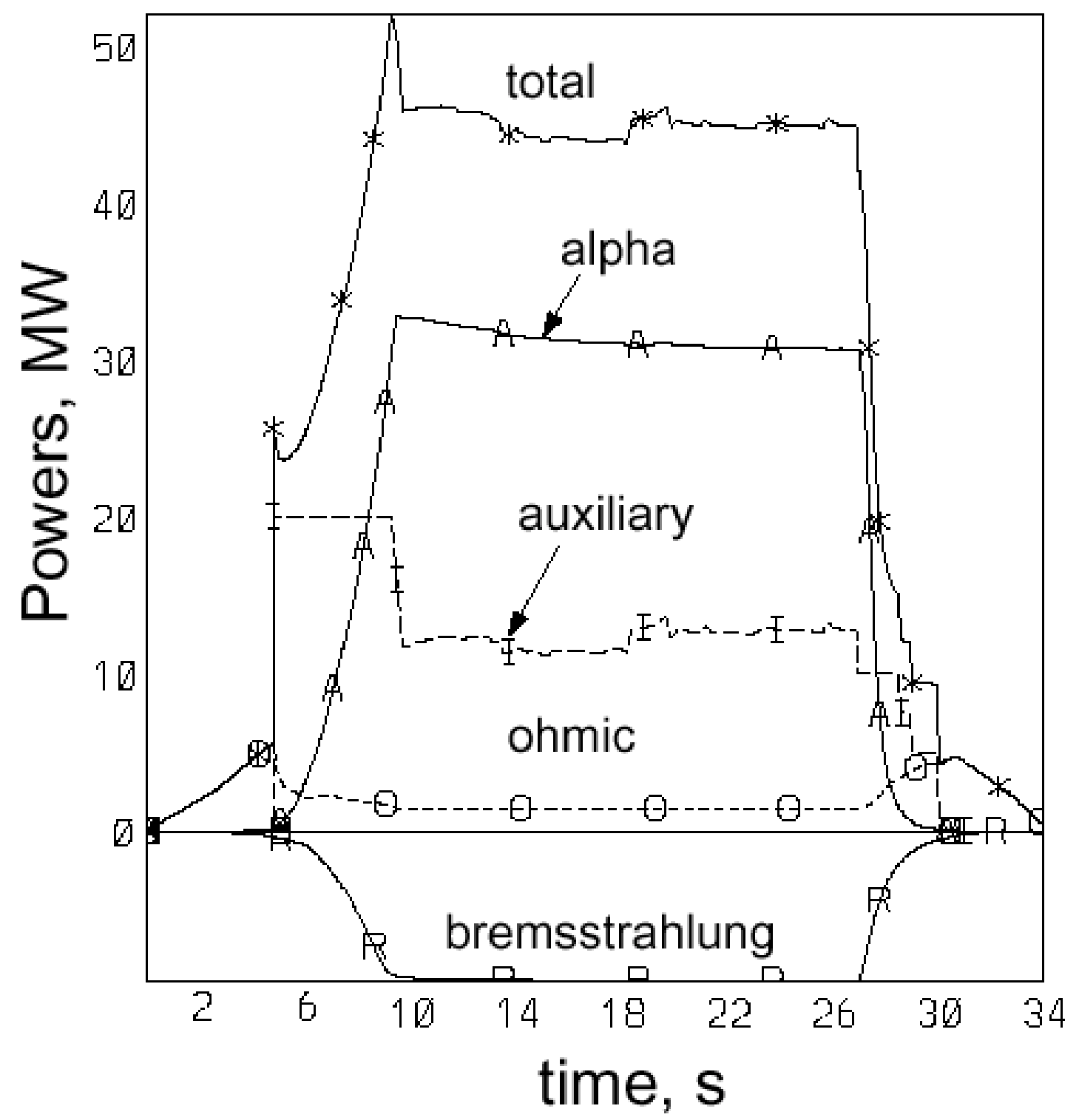

Figure 4: Time histories of the various powers into and lost from the plasma for the FIRE reference discharge. 

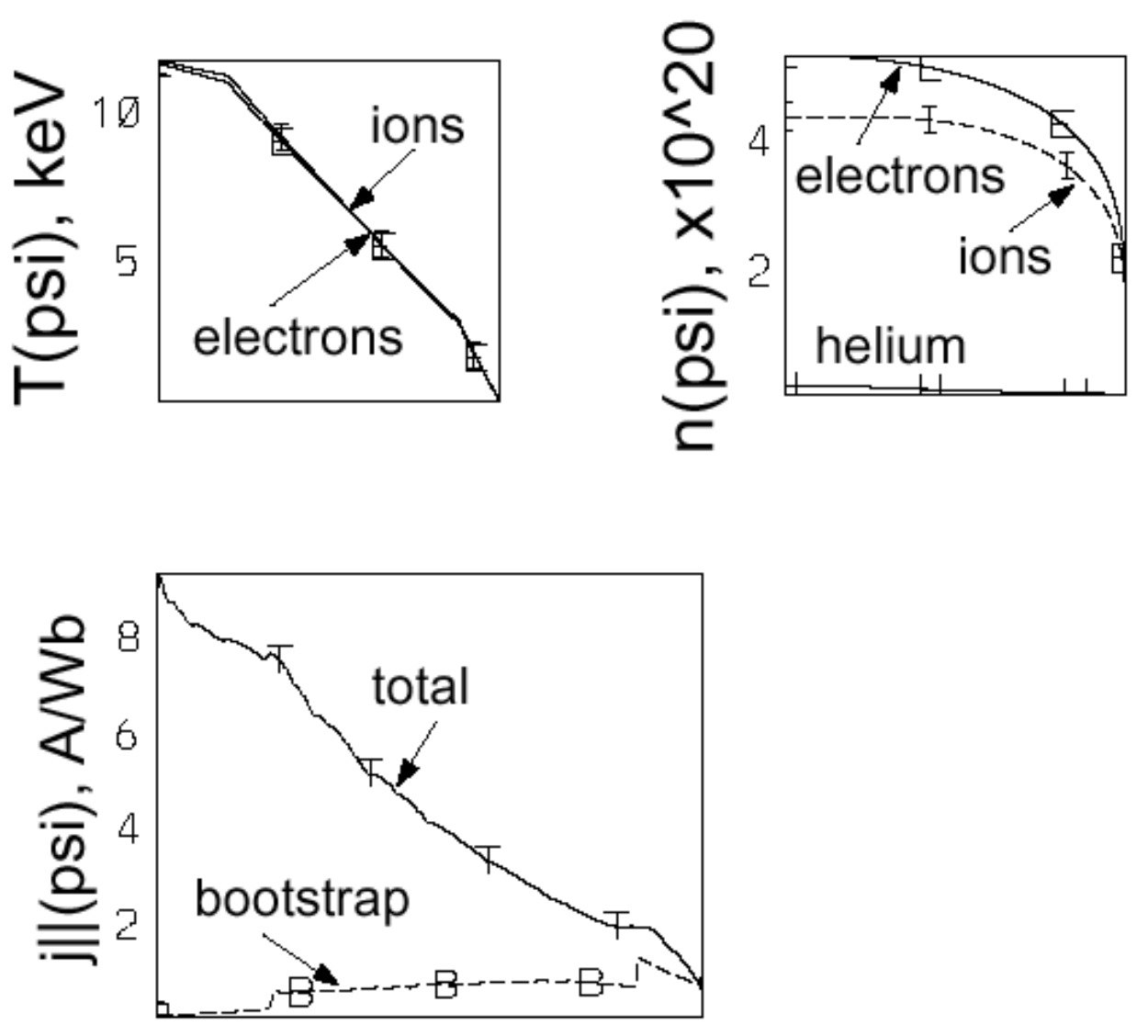

Figure 5: Profiles of the electron and ion temperatures and densities, and the plasma current density during the flattop for the FIRE reference discharge. 


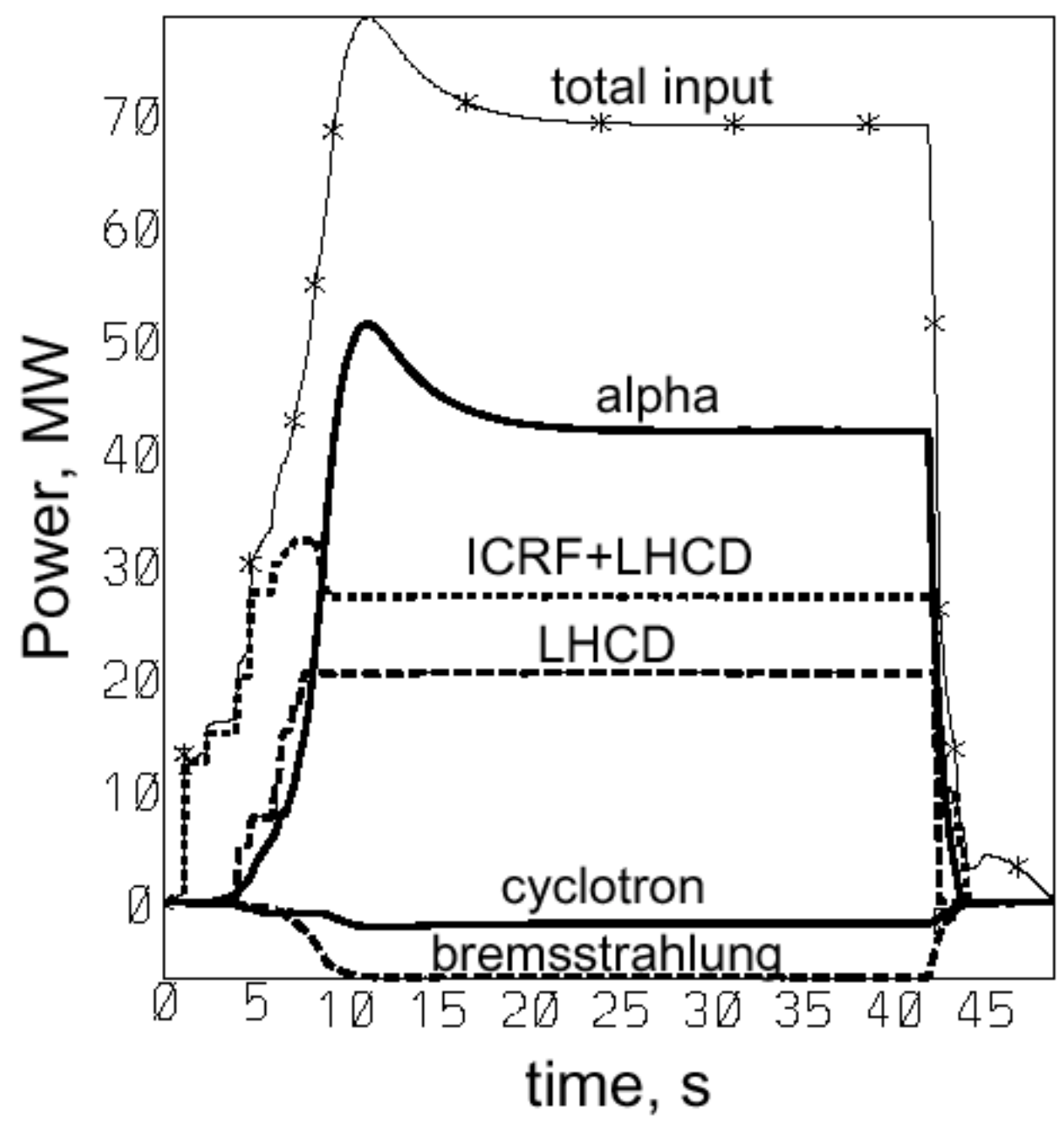

total non-inductive

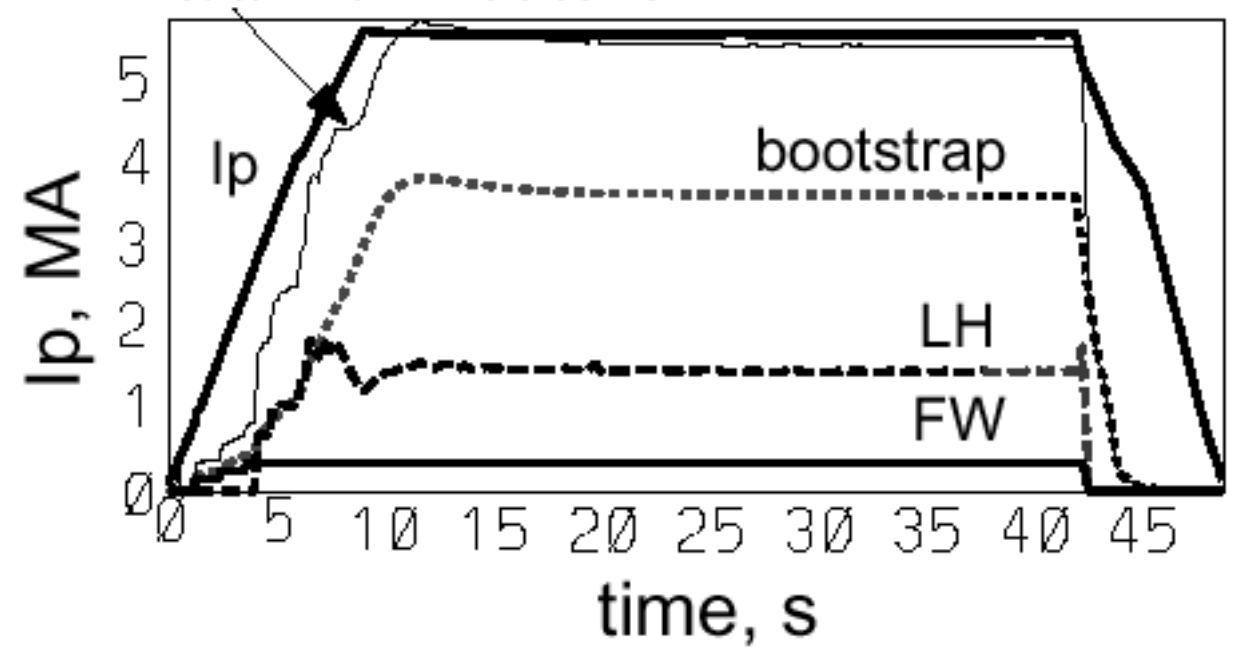

Figure 6: Time histories of the contributions to the plasma current, and various powers into and lost from the plasma for an AT scenario in FIRE. 

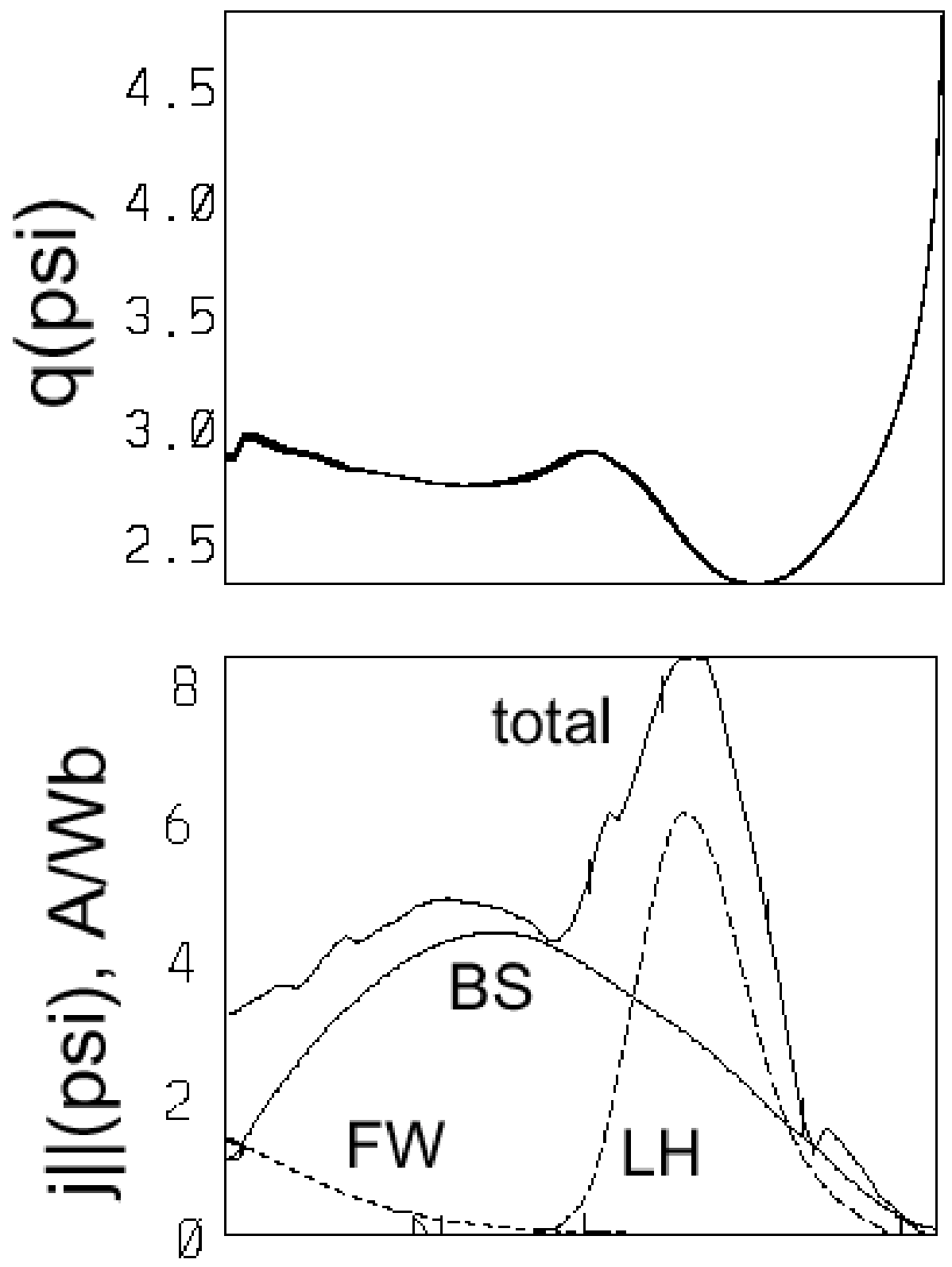

Figure 7: Plasma safety factor and current density during flattop for an AT scenario in FIRE. 


\section{External Distribution}

Plasma Research Laboratory, Australian National University, Australia

Professor I.R. J ones, Flinders University, Australia

Professor J oão Canalle, Instituto de Fisica DEQ/IF - UERJ , Brazil

Mr. Gerson O. Ludwig, Instituto Nacional de Pesquisas, Brazil

Dr. P.H. Sakanaka, Instituto Fisica, Brazil

The Librarian, Culham Laboratory, England

Library, R61, Rutherford Appleton Laboratory, England

Mrs. S.A. Hutchinson, JET Library, England

Professor M.N. Bussac, Ecole Polytechnique, France

Librarian, Max-Planck-Institut für Plasmaphysik, Germany

J olan Moldvai, Reports Library, MTA KFKI-ATKI, Hungary

Dr. P. Kaw, Institute for Plasma Research, India

Ms. P.J . Pathak, Librarian, Insitute for Plasma Research, India

Ms. Clelia De Palo, Associazione EURATOM-ENEA, I taly

Dr. G. Grosso, Instituto di Fisica del Plasma, Italy

Librarian, Naka Fusion Research Establishment, J AERI, J apan

Library, Plasma Physics Laboratory, Kyoto University, J apan

Research Information Center, National Institute for Fusion Science, J apan

Dr. O. Mitarai, Kyushu Tokai University, J apan

Library, Academia Sinica, Institute of Plasma Physics, People's Republic of China

Shih-Tung Tsai, Institute of Physics, Chinese Academy of Sciences, People's Republic of China

Dr. S. Mirnov, TRINITI, Troitsk, Russian Federation, Russia

Dr. V.S. Strelkov, Kurchatov Institute, Russian Federation, Russia

Professor Peter Lukac, Katedra Fyziky Plazmy MFF UK, Mlynska dolina F-2, Komenskeho Univerzita, SK-842 15 Bratislava, Slovakia

Dr. G.S. Lee, Korea Basic Science Institute, South Korea

Mr. Dennis Bruggink, Fusion Library, University of Wisconsin, USA

Institute for Plasma Research, University of Maryland, USA

Librarian, Fusion Energy Division, Oak Ridge National Laboratory, USA

Librarian, Institute of Fusion Studies, University of Texas, USA

Librarian, Magnetic Fusion Program, Lawrence Livermore National Laboratory, USA

Library, General Atomics, USA

Plasma Physics Group, Fusion Energy Research Program, University of California at San Diego, USA

Plasma Physics Library, Columbia University, USA

Alkesh Punjabi, Center for Fusion Research and Training, Hampton University, USA

Dr. W.M. Stacey, Fusion Research Center, Georgia Institute of Technology, USA

Dr. J ohn Willis, U.S. Department of Energy, Office of Fusion Energy Sciences, USA

Mr. Paul H. Wright, Indianapolis, Indiana, USA 
The Princeton Plasma Physics Laboratory is operated by Princeton University under contract with the U.S. Department of Energy.

\author{
Information Services \\ Princeton Plasma Physics Laboratory \\ P.O. Box 451 \\ Princeton, NJ 08543
}

Phone: 609-243-2750

Fax: 609-243-2751

e-mail: pppl_info@pppl.gov

Internet Address: http://www.pppl.gov 